
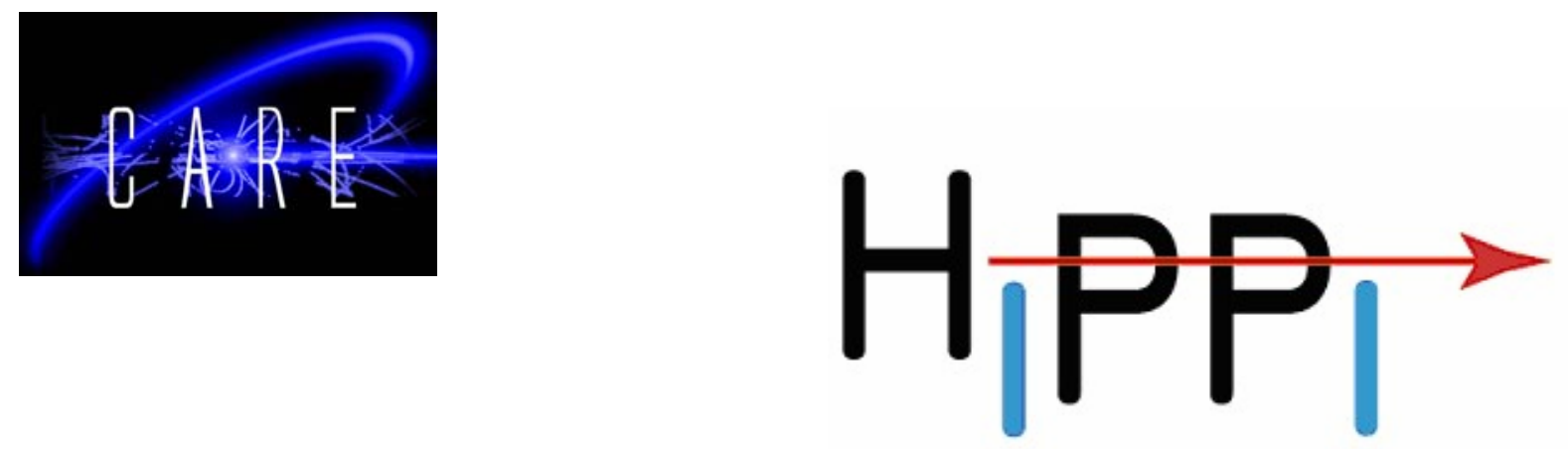

\title{
DEVELOPMENT OF A NORMAL CONDUCTING CH-DTL
}

G. Clemente, H. Podlech, R. Tiede, U. Ratzinger, IAP, Frankfurt, Germany

L. Groening, GSI, Darmstadt, Germany. S. Minaev, ITEP, Moscow, Russia

\begin{abstract}
The normal conducting "Crossbar H-Type" $(\mathrm{CH})$ accelerating structure is a good candidate for pulsed, high intensity linacs application, covering the energy range from 3 to $100 \mathrm{MeV}$. $\mathrm{H}$ Mode cavities are outstanding in the low-beta range with respect to shunt impedance, high acceleration fields, and compact design: that's why we propose to base the $70 \mathrm{~mA}, 70 \mathrm{MeV}$, $352 \mathrm{MHz}$ proton linac for the GSI FAIR project on that structure. The actual design consists of $11 \mathrm{CH}-\mathrm{DTL}$ 's with a total length of around $20 \mathrm{~m}$. Latest results from beam dynamics optimisation will be discussed. Moreover, this paper describes the CH-DTL cavity design with emphasis on the optimisation with Microwave Studio and on the achieved progress in the development of mechanical design concepts. A stainless steel multicell model cavity is presently fabricated by our institute in collaboration with GSI, in order to investigate manufacturing and assembly details. Based on this experience, the design of a $\mathrm{CH}$ prototype power cavity will be optimised.
\end{abstract}

Contribution to the PAC05, Knoxville, USA

Work supported by the European Community-Research Infrastructure Activity under the FP6 "Structuring the European Research Area" programme (CARE, contract number RII3-CT2003-506395). 


\title{
DEVELOPMENT OF A NORMAL CONDUCTING CH-DTL*
}

\author{
G. Clemente, H. Podlech, R. Tiede, U. Ratzinger, IAP, Frankfurt, Germany \\ L. Groening, GSI, Darmstadt, Germany. \\ S. Minaev, ITEP, Moscow, Russia
}

\begin{abstract}
The normal conducting "Crossbar H-Type" (CH) accelerating structure is a good candidate for pulsed, high intensity linacs application, covering the energy range from 3 to $100 \mathrm{MeV}$. H Mode cavities are outstanding in the low-beta range with respect to shunt impedance, high acceleration fields, and compact design: that's why we propose to base the $70 \mathrm{~mA}, 70 \mathrm{MeV}, 352 \mathrm{MHz}$ proton linac for the GSI FAIR project on that structure. The actual design consists of $11 \mathrm{CH}-\mathrm{DTL}$ 's with a total length of around $20 \mathrm{~m}$. Latest results from beam dynamics optimisation will be discussed. Moreover, this paper describes the CH-DTL cavity design with emphasis on the optimisation with Microwave Studio and on the achieved progress in the development of mechanical design concepts. A stainless steel multicell model cavity is presently fabricated by our institute in collaboration with GSI, in order to investigate manufacturing and assembly details. Based on this experience, the design of a $\mathrm{CH}$ prototype power cavity will be optimised.
\end{abstract}

\section{BEAM DYNAMICS INVESTIGATION}

Compared to previous design results for the GSI FAIR facility Proton Linac [1], the RFQ-DTL matching section (MEBT) has been modified, according to a new RFQ output particle distribution at energy of $3 \mathrm{MeV}$ and with a current higher than $70 \mathrm{~mA}$.

\section{The proposed MEBT Section}

The MEBT section (represented in Fig.1) must provide efficient matching between the RFQ and the first $\mathrm{CH}$ tank and also provides some free space for the beam diagnostics devices like phase probes, profile monitors or current transformer: for this reason the proposed scheme includes some free space followed by a single quadruple lens, a short rebuncher and, finally, a triplet system that leads the beam into the first $\mathrm{CH}$ module. Nevertheless this first stage of acceleration is still under investigation: it could be happen than more free space is needed and this could consider influence the transversal behaviour of the beam. Preliminary results have shown that, for a drift length higher than $15 \mathrm{~cm}$ the initial singlet could be replaced by a doublet quadruple system in order to provide transversal stability and symmetry of the beam.

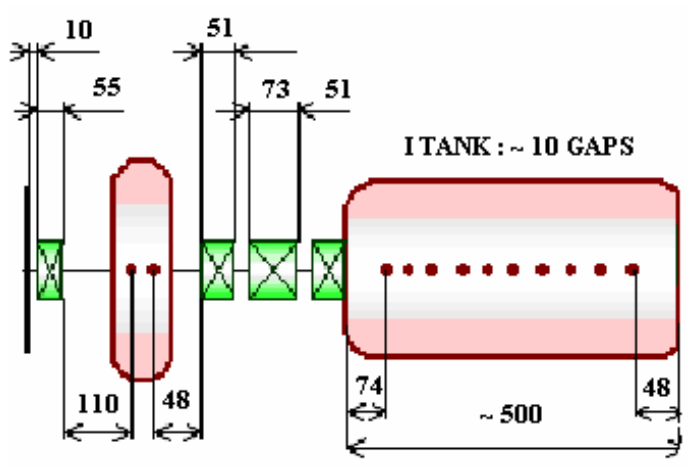

Fig.1: a schematic overview of the MEBT section showing the rebuncher and the first tank.

\section{The overall design}

After the rebuncher 10 tanks (for a total length of $\sim 20$ meters) will be needed to get the final energy of $70 \mathrm{MeV}$ within the beam requirements for the injection into the SIS Synchrotron [1]

Each tank corresponds to a KONUS [2] period and will be fed by its own $352 \mathrm{MHz}$ Klystron with a maximum power of 1.1 MW: to keep the total power consumption per cavity (energy absorbed by the beam + loss due to the electrical resistance) below this number the effective shunt impedance has been optimised and a value of 35 $\mathrm{M} \Omega / \mathrm{m}$ seems feasible at $70 \mathrm{MeV}$ [3].

Finally a new concept for the hosting of the quadruple triplets between each tank has been developed and it will be described in the next section.

The main $\mathrm{CH}$ parameters are summarized in Tab.1 while Figg. 2 and 3 show, respectly, the transverse envelopes and the input and output projection emittance cluster plots. 


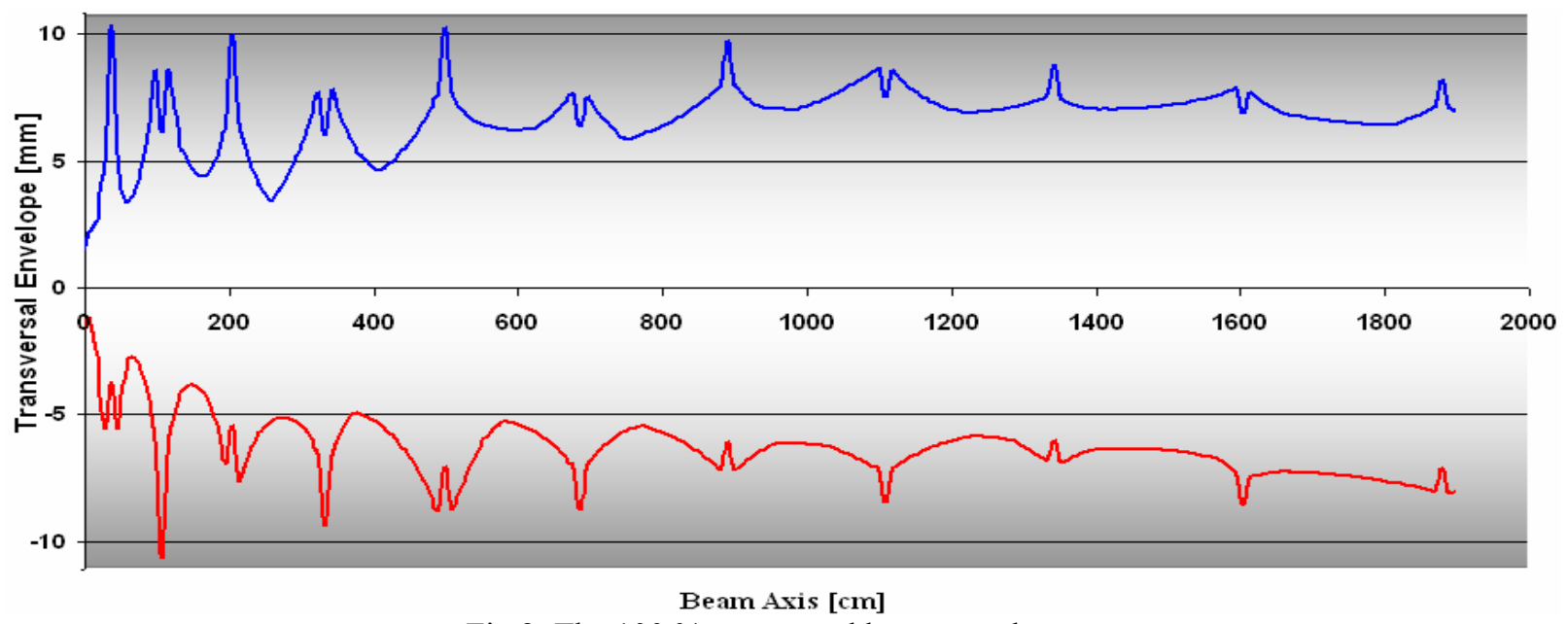

Fig.2: The $100 \%$ transversal beam envelope
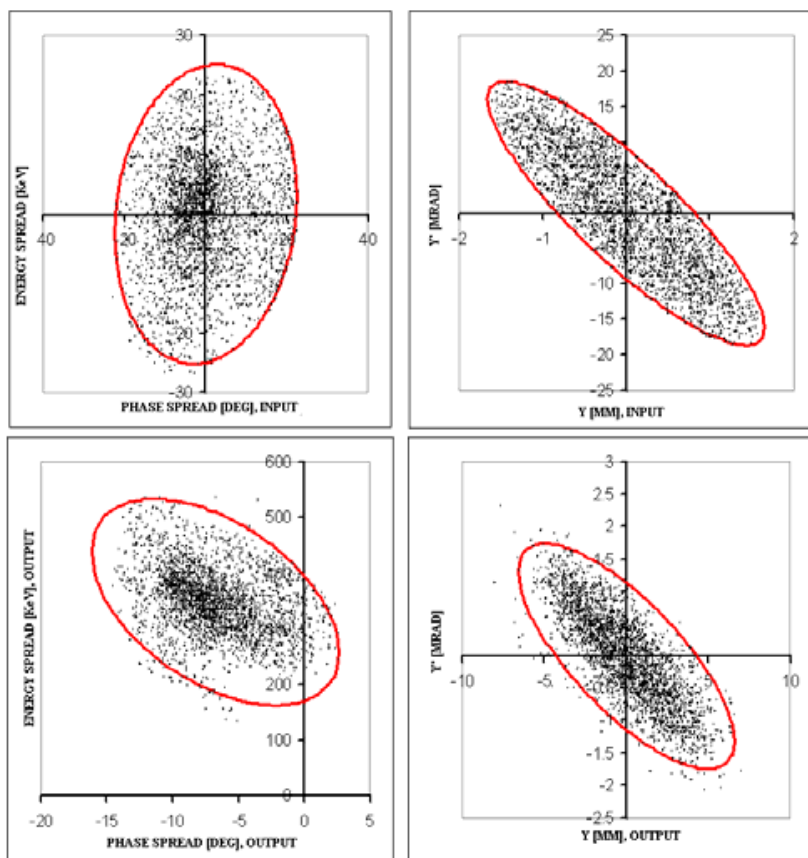

Fig.3: Transverse and longitudinal particle distribution at the entrance and at the exit of the CH-DTL.

Tab. 1: The main parameters of the CH-DTL

\begin{tabular}{|c|c|c|}
\hline CH Parameters & INPUT & OUTPUT \\
\hline Energy [MeV] & 3 & 70 \\
\hline Current [mA] & 95.7 & 95.7 \\
\hline$\varepsilon_{\text {tr }}(98 \%$ norm) & 1.2266 & 2.9033 \\
\hline$\varepsilon_{\text {tr }}($ RMS) & 0.23768 & 0.37551 \\
\hline$\varepsilon_{\text {long }}(98 \%$ norm) & 4.4252 & 12.058 \\
\hline$\varepsilon_{\text {long }}($ RMS) & 0.6752 & 1.4244 \\
\hline No. of Tanks & \multicolumn{2}{|c|}{10} \\
\hline No. of gaps per tank & \multicolumn{2}{|c|}{17} \\
\hline Total Length [meter] & \multicolumn{2}{|c|}{$\sim$} \\
\hline
\end{tabular}

\section{MECHANICAL DEVELOPMENT}

There is still a lack in experience with this novel type of accelerator: for this reason we decided to build a cold model to test the fabrication steps and to find out technical solutions for the future prototype cavity.

Our model consists of 8 identical cells corresponding to a beta value of $0.09(\beta \lambda / 2=45 \mathrm{~mm})$ which, according to beam dynamics simulation, would be the length of the last gap in the first module. In a first step Microwave Studio ${ }^{\circledR}$ was used to optimize cavity parameters such us shunt impedance and voltage distributions: comparison between different geometries for the stems were carried out both at the low and high energy front side and, finally a conical solution for the stems was adopted. The dimensions were finally optimised to take into account the space needed for the water cooling system and to ensure for the whole structure a good mechanical stability.

As already discussed in [4], the stems will be welded in the outer wall while the massive copper half drift tubes will be press-fit into the stainless steel stems: this technique is expected to ensure both excellent RF contacts and mechanical stability. At the moment no screws are foreseen inside the cavity with the aim to improve the electrical properties of the cavity.

One of the main advantages of the new CH-DTL, also according to the experience on the superconducting model [5], is the capability to get flatness of the field distribution: this is especially important in the ends cell to approximate the $\mathrm{H}_{211}$ mode as close as possible to the $\mathrm{H}_{210}$; in the $\mathrm{IH}$ cavity $\left(\mathrm{H}_{110}\right.$ mode), for instance, this is obtained by large undercuts in the base girders to enlarge the magnetic inductance at the cavity ends. In our case, since no girder is present in the cavity, one way is to increase the length of the first and last half tubes: if the radius of these half tubes is also enlarged, this drift space can be used to host one part of the quadruple lens or a diagnostics device. For our model we tested the distribution of the field obtained for a end cell length of 8 , 10 and $12 \mathrm{~cm}$, respectively, and the results are shown in Fig. 4 where it is possible to see how good flatness of the eclectic field and voltage distribution can be achieved 
simply by using this technique: further improvement can, of course, be obtained by changing the drift tube length inside the desired gap.

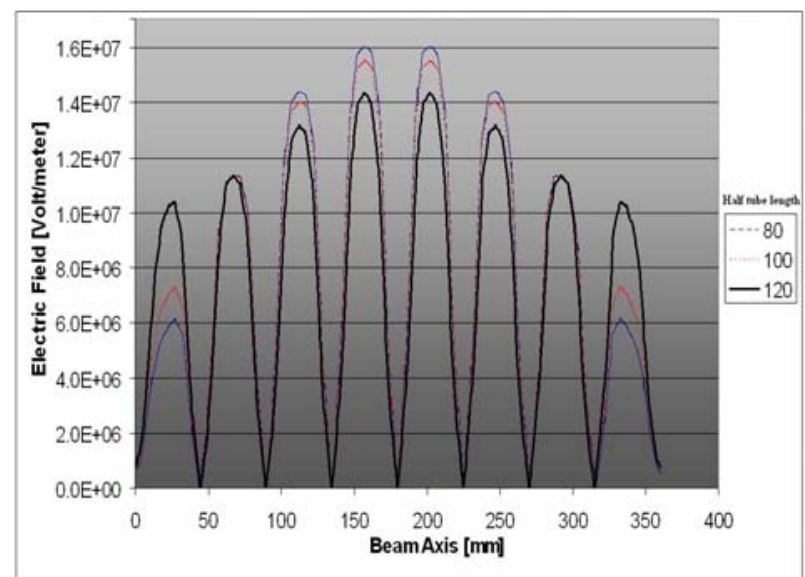

Fig.4: the Field distribution for different value of the end half tube length.

But this design will not be only helpful for the cavity tuning, it will carry also great advantages concerning the mechanical assemblage: hosting the lenses and the diagnostics inside the end cells will permit to save considerable quantity of space resulting in a huge reduction of the total size of the linac. Each tank will be fed by its own generator and will be strictly integrated with the following one, resulting in a very compact structure considerably improving the CH-DTL capabilities. Least, the reduction of the drift space between one tank and the following one will also be useful concerning the particles transport.

\section{CONCLUSION}

A compact design of a dedicated Proton Linac, based on $\mathrm{H}$-mode cavities $(\mathrm{CH})$ for the FAIR facility at GSI was presented. Preliminary beam dynamics simulations show that the beam could be transported from 3 to $70 \mathrm{MeV}$ within the requirements for the SIS Injection. A new design for the cavity ends cell, which will considerably improve the CH-DTL capabilities, was described.

According to this design a cold model is under construction at the IAP in order to test key steps in production and assemblage.

\section{REFERENCE}

[1] R. Tiede and Al, "KONUS Beam Dynamics Design of a $70 \mathrm{MA}, 70 \mathrm{MEV}$ Proton CH-DTL for GSI-SIS12", LINAC '04, Lubeck, pag. 60.

[2] U. Ratzinger and R. Tiede, "Status of the HIIF RF linac study based on H-mode cavities", Nucl. Instr. and Meth. in Phys. Res. A 415 (1998), pp. 229-235.

[3] Z. Li et al., "Design of the R.T. CH-Cavity and Perspectives for a New GSI Proton Linac", LINAC '04, Lubeck, pag. 81

[4] G. Clemente et Al, "Geometrical optimisation of the $70 \mathrm{~mA}, 70 \mathrm{MeV}$ CH-Proton Injector Cavity for the FAIR Project”, GSI Annual Report 04

[5] H. Podlech et Al, "Development of Superconducting $\mathrm{CH}$ Structure for low and mediaum beta beams and the status of the $352 \mathrm{MHz}$ Cavity", ICFA-HB 2004

Fig.5: Overview of the proposed design for the CH-DTL: the large end cells will host the quadrupole lenses and the beam diagnostics while the massive copper drift tubes (shown here in white) will be press-fit into the stainless steel

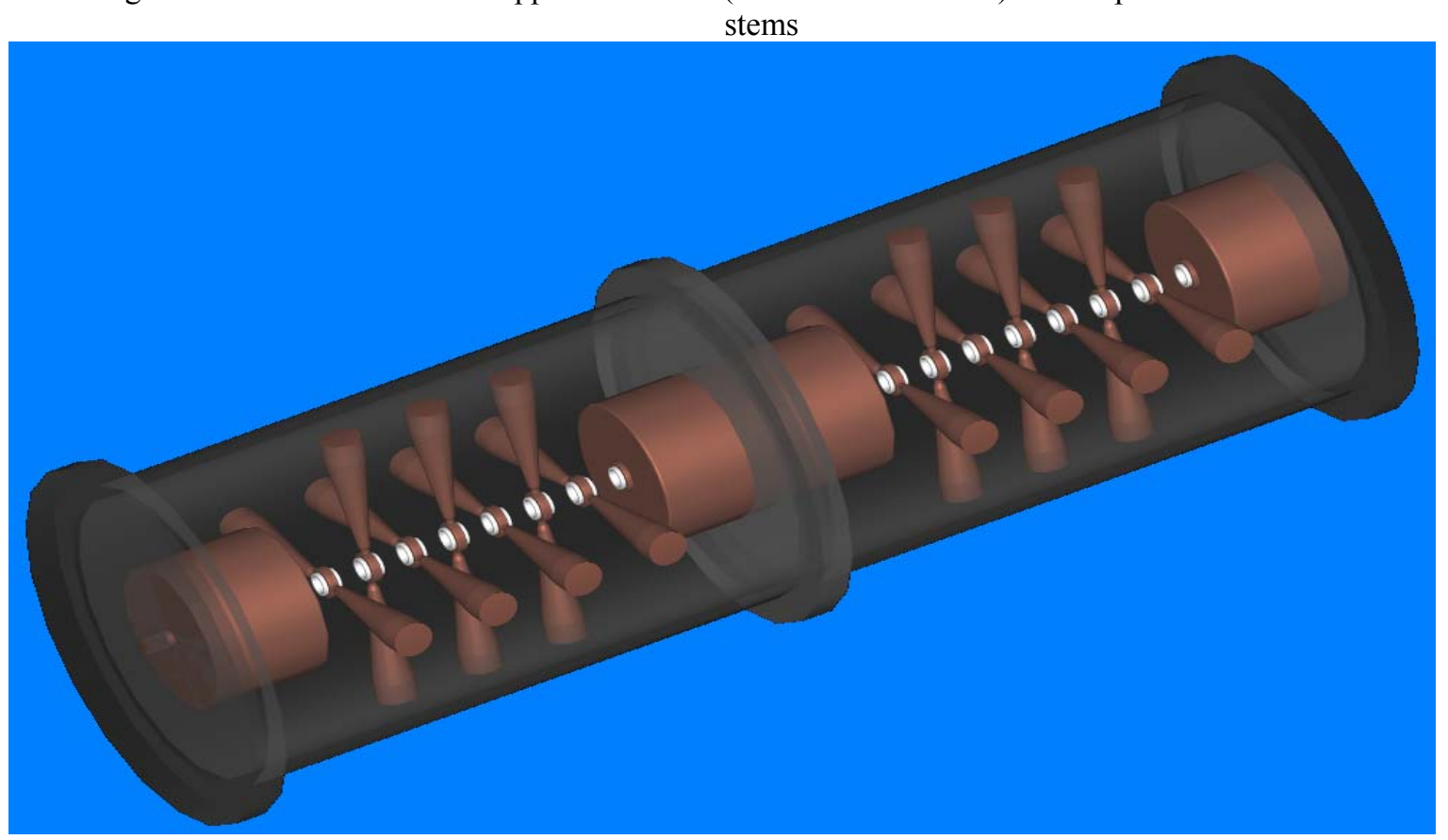

